Portland State University

PDXScholar

8-1-1968

\title{
Solving random walk problems using resistive analogues
}

Richard D. Morris

Portland State University

Follow this and additional works at: https://pdxscholar.library.pdx.edu/open_access_etds Let us know how access to this document benefits you.

\section{Recommended Citation}

Morris, Richard D., "Solving random walk problems using resistive analogues" (1968). Dissertations and Theses. Paper 529.

https://doi.org/10.15760/etd.529

This Thesis is brought to you for free and open access. It has been accepted for inclusion in Dissertations and Theses by an authorized administrator of PDXScholar. Please contact us if we can make this document more accessible: pdxscholar@pdx.edu. 
AN ABSTRACT OF THE THESIS OF Richard D. Morris for the Master of Science in Applied Science presented August 8, 1968.

Title: Solving Random Walk Problens Using Resistive Analogues.

APPROVED BY MEMBERS OF THE THESIS COMNITTEE:

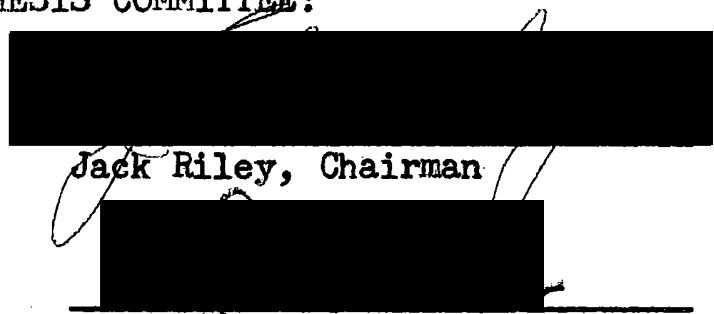

Selmo Tąuber

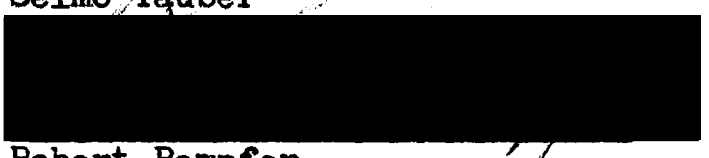

Robert Renpfer 
The classical method of solving random walk problems involves using Markov chain theory. When the particular random walk of interest is written in matrix form using Markov chain theory, the problem must then be solved using a digital computer. To solve all but the most trivial random walk problems by hand would be extremely difficult and time consuming. Very large random walk problems may even prove difficult to solve on the smaller digital computers.

This paper intends to demonstrate a method that may be used to solve large random walk problems in a quick and economical manner. This alternate method uses resistive analogues and has the added feature of extracting particular solutions without having to completely solve the problem as would be necessary using a digital computer. Many analogues of random walks may also be quickly amended to include other random walks with relative ease using this alternate method of solution. Because this method uses nothing more than a power supply, a DC voltmeter and a set of resistors, the analogue of, a particular random walk problem may be left set-up without incurring any loss of time or money on a digital computer. Once the resistors are mounted in a permanent fashion, the random walk analogues may also be used as an effective demonstration of random walk probabilities in the classroom. 
SOLVING RANDOM WALK PROBLEMS USING RESISTIVE ANALOGUES

by

RICHARD MORRIS

MASTER'S THESIS

APPLIED SCIENCE

SUMMER 1968

PORTLAND STATE COLLEGE 
TO THE OFFICE OF GRADUATE STUDIES:

The members of the Committee approve the thesis of

Richard D. Morris presented August 8, 1968.

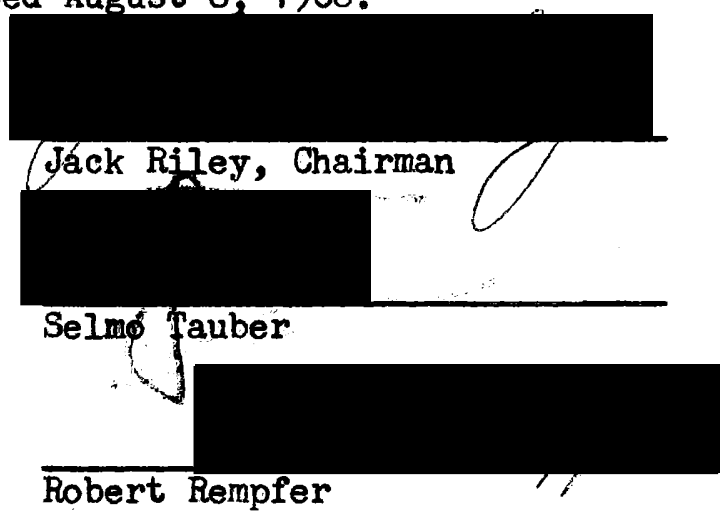

APPROVED :

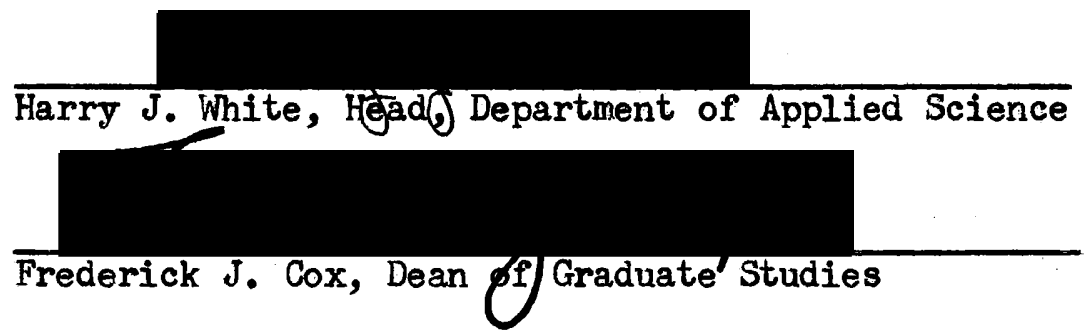


$-i-$

-- ACKNOWLEDGMENT --

The writer wishes to express his appreciation for the many helpful suggestions made by Dr. Robert Rempfer in the preparation of this paper. 
Finite Markov Chains 2

Finite Absorbing Markov Chains 5.

Finite Absorbing Random Walks 14

Electric Circuits 18

Resistive Analogues 21.

Solved Random Walks 25

Bibliography 41 
SOLVING RANDOM WALK PROBLEMS USING RESISTIVE ANALOGUES

The classical method of solving random walk problems involves using Markov chain theory. When the particular random walk of interest is written in matrix form using Markov chain theory, the problem must then be solved using a digital computer. To solve all but the most trivial random walk problems by hand would be extremely difficult and time consuming. Very large random walk problems may even prove difficult to solve on the smaller digital computers.

This paper intends to demonstrate a method that may be used to solve large random walk problems in a quick and economical manner. This alternate method uses resistive analogues and has the added feature of extracting particular solutions without having to completely solve the problem as would be necessary using a digital computer. Many analogues of random walks may also be quickly amended to include other random walks with relative ease using this alternate method of solution. Because this method uses nothing more than a power supply, a DC voltmeter and a set of resistors, the analogue of a particular random walk problem may be left set-up without incurring any loss of time or money on a digital computer. Once the resistors are mounted in a permanent fashion, the random walk analogues may also be used as an effective demonstration of random walk probabilities in the classroom.

To understand the random walk problem and its solution, it is first necessary to review Markov chain theory. By using Markov chain theory, the equivalence of resistive networks to random walks can be demonstrated. A knowledge of basic probability, matrix and DC circuit theory is also 
necessary and will be assumed.

-- FINITE MARKOV CHAINS --

A Markov chain is an important type of stochastic process. A stochastic process is any sequence of events or experiments that can be subjected to a probabilistic analysis. If the set of possible outcomes is finite, the process is said to be finite.

DEFINITION. A finite stochastic process with outcome functions $f_{0}, f_{1}$, ..., $f_{n}$ is a Markor chain process if the starting state, given by $f_{0}$, is fixed and

$$
\begin{aligned}
& \operatorname{Pr}\left[f_{n}=t /\left(f_{n-1}=s\right) \wedge\left(f_{n-2}=r\right) \wedge \cdots \wedge\left(f_{1}=a\right)\right] \\
& =\operatorname{Pr}\left[f_{n}=t \mid f_{n-1}=s\right] \\
& \operatorname{Pr}\left[f_{n}=t \mid f_{n-1}=s\right]=\operatorname{Pr}\left[f_{m}=t / f_{m-1}=s\right]
\end{aligned}
$$

for all $m \geq 1, n \geq 2$ and any possible sequence of outcomes $a, \ldots, s, t$.

In other words, the outcome of a given event depends only on the outcome of the immediately preceding event; and, moreover, this dependence is the same at all stages. A Markov chain process may also be defined using another, equivalent, definition.

DEFINITION. A Markov chain process is determined by specifying the following information: There is a given set of states $\left\{s_{1}, s_{2}, \ldots, s_{r}\right\}$. The process can be in one and only one of these states at a given time and it moves successively from one state to another. Each move is 
called a step. The probability that the process moves from $s_{1}$ to $s_{\mathrm{f}}$ depends only on the state $s_{i}$ that it occupied before the step. The transition probability $p_{i j}$, which gives the probability that the process will move from $s_{i}$ to $s_{j}$, is given for every ordered pair of states. Also, an initial starting state is specified at which the process is assumed to begin. 1

Given the set of transition probabilities $\left\{p_{i j}\right\}$, these probabilities can be arranged in the form of a transition matrix $P$,

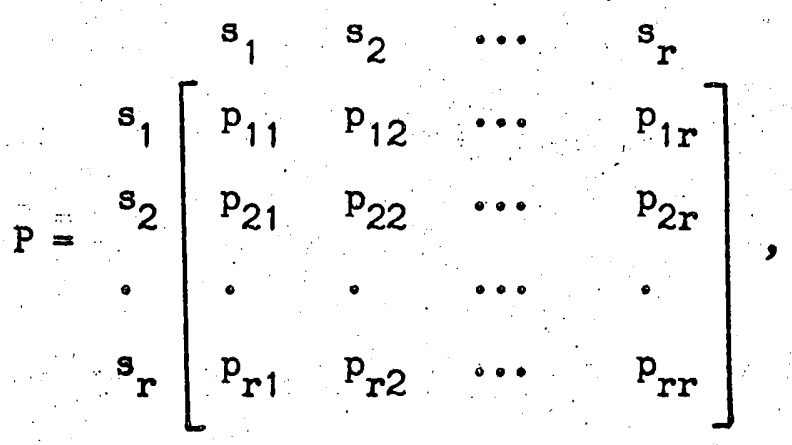

where each row of $P$ is a probability vector with the following properties.

(3)

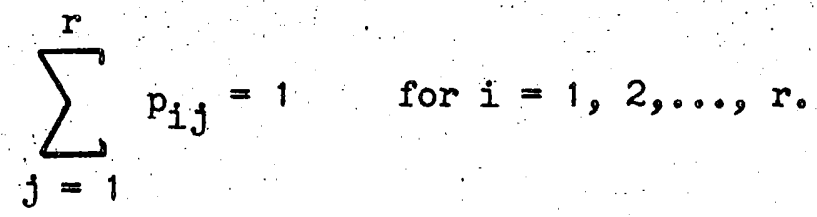

(4)

$$
p_{i j} \geq 0 \text { for all } i, j \text {. }
$$

A Markov chain can now be determined completely by specifying the 
transition matrix $P$ and the starting state.

Once the Markov chain is determined, the outcome probabilities of the $n$-th step can be analyzed. Given the set of states $\left\{s_{1}, s_{2}, \ldots, s_{r}\right\}$, a new weight function $w^{(n)}$, corresponding to the $n-t h$ step, can be assigned to each state. The new weight functions for each state will be

$$
w^{(n)}\left(s_{j}\right)=\operatorname{Pr}\left[f_{n}=s_{j}\right]
$$

All possible $n$-th outcome weights can then be expressed by the vector $\vec{W}^{(n)}$ as

$$
\vec{w}^{(n)}=\left(w^{(n)}\left(s_{1}\right), w^{(n)}\left(s_{2}\right), \ldots, w^{(n)}\left(s_{r}\right)\right)
$$

Given the $W^{(n)}$ vector, it is possible to determine the $W^{(n+1)}$ vector using the finite case of the Chapman - Kolmogorov equation. This equation is

$$
w^{(n+1)}\left(s_{j}\right)=\sum_{k=1}^{r} w^{(n)}\left(s_{k}\right)^{\prime} p_{k j}
$$

In matrix language, this is

$$
\vec{W}^{(n+1)}=\vec{W}^{(n)} \cdot P \text {. }
$$

Thus, the vector giving the probabilities for being in each of the states after $n+1$ steps is obtained by multiplying the transition matrix on the left by the probability vector corresponding to $n$ steps. If the process 
is started in $s_{i}, \vec{W}^{(1)}$ will be the i-th row of $P, \vec{W}^{(2)}=\vec{W}^{(1)} \cdot P$, $\vec{W}^{(3)}=\vec{W}^{(2)} \cdot \dot{P}, \ldots$, etc.

The probabilities for being in each of the states after in steps can also be determined by performing operations on the transition matrix $P$. If the process is started in $s_{i}$ and $\vec{W}^{(1)}$ is the $i-$ th row of $P$, then $\vec{W}^{(2)}=\vec{W}^{(1)} \cdot \mathrm{P}$ is the $i-t h$ row of $P^{2}, \vec{W}^{(2)} \cdot P$ is the $i-t h$ row of $P^{3}, \ldots$ -, etc. Hence $\vec{W}^{(n)}$ is the $i-$ th row of $P^{n}$. Thus, the various rows of $\mathrm{P}^{\mathrm{n}}$ give the vector $\vec{W}^{(n)}$ for various starting states. The probability that the process will be in state $s_{j}$ after $n$ steps if it is started in state $s_{i}$, can now be expressed as $p_{i j}^{(n)}$, which is given by the $i j-t h$ entry of the matrix $P^{n}$.

\section{-- FINITE ABSORBING MARKOV CHAINS --}

A special class of Markov chains called absorbing Markov chains will now be introduced.

DEFINITION. A state in a Markov chain is an absorbing state if it is impossible to leave it. A Markov chain is absorbing if (1) it has at least one absorbing state, and (2) from every state it is possible to go to an absorbing state (not necessarily in one step).

When a process reaches an absorbing state, the process is said to be absorbed. If the k-th state is an absorbing state, then the k-th row of the matrix $P$ will have the probabilities 
(5) $\quad \mathrm{p}_{\mathrm{kk}}=1$.

(6) $\quad \mathrm{p}_{\mathrm{km}}=0 \quad$ for $\mathrm{m} \neq \mathrm{k}$.

The three most pertinent questions concerning finite absorbing Markov chains are:

1. What is the probability that the process will be absorbed In a given state?

2. On the average, how long will it take for the process to be absorbed?

3. On the average, how many times will the process be in each nonabsorbing state?

The following theorems and definitions will be needed to answer these questions.

1.0 THEOREM. In a finite absorbing Markov chain, no matter where the process starts, the probability after $n$ steps that the process is in an absorbing state tends to 1 as $n$ tends to infinity.

PROOF. By definition, from every state it is possible to go to an absorbing state. Suppose that from any nonabsorbing state it is possible to reach an absorbing state in not more than $n$ steps. Further, let $n$ be the largest of the number of steps required from each state. Hence there is a positive number $p$ such that the probability of entering an absorbing state in at most $\mathrm{n}$ steps is at least $\mathrm{p}$, from every nonabsorbing state. Hence the probability of not reaching an absorbing state in $n$ steps is 
at most $(1-p)$, which is less than 1. The probability of not reaching an absorbing state in $k n$ steps is less than or equal to $(1-p)^{k}$, and this probability tends to 0 as $k$ increases. Hence the theorem follows.

Consider, now, an arbitrary finite absorbing Markov chain. The states will be renumbered so that the absorbing states come first. If there are $s$ nonabsorbing states and $r-s$ absorbing states, the transition matrix will have the following canonical form.

$$
\left.P=\left[\begin{array}{ccc}
I & s & 0 \\
\hdashline-s & -m \\
R & 0
\end{array}\right]\right\} s
$$

Here $I$ is an $(r-s) \times(r-s)$ identity matrix, 0 is an $(r-s) \times s$ zero matrix, $R$ is an $s \times(r-s)$ matrix and $Q$ is an $s \times s$ matrix.

Earlier, it was shown that the various rows of $\mathrm{P}^{\mathrm{n}}$ give the vector $W^{(n)}$, which corresponds to the various weight functions for each state after $n$ steps. If $P$ is multiplied by itself $n$ number of times observing the partition, the matrix $\mathrm{P}^{\mathrm{n}}$ will be 


$$
\begin{aligned}
& -8- \\
& P^{n}=\left[\begin{array}{c:c}
I & 1 \\
\hdashline-1 & 0 \\
\hdashline R^{*} & Q^{n}
\end{array}\right]
\end{aligned}
$$

From Theorem 1.0 it can be seen that the powers of $Q$ tend to 0 . Hence as $P$ is raised to higher and higher powers, the partioned matrices approach a matrix whose last s columns are all 0 . This is the matrix version of Theorem 1.0.

1.1 THEOREM. For any finite absorbing Markov chain, I-Q has an inverse, and

$$
(I-Q)^{-1}=I+Q+Q^{2}+\cdots=\sum_{k=0}^{\infty} Q^{k}
$$

PROOF. Consider the identity

$$
(I-Q) \cdot\left(I+Q+Q^{2}+\cdots+Q^{n-1}\right)=I-Q^{n} \cdot
$$

As a consequence of Theorem 1.0, the right side tends to I. This matrix has determinant 1. Hence for sufficiently large $n$, I- $Q^{n}$ must have a non-zero determinant. But the determinant of a product of 
two matrices is the product of the determinants, hence I-Q cannot have a zero determinant. The determinant not being equal to zero is a sufficient condition for a matrix to have an inverse. Hence $I-Q$ has an inverse. Since this inverse exists, it is possible to multiply both sides of the identity by it:

$$
I+Q+Q^{2}+\cdots+Q^{n-1}=(I-Q)^{-1} \cdot\left(I-Q^{n}\right)
$$

But the right side of this new identity clearly tends to $(I-Q)^{-1}$, which completes the proof. ${ }^{2}$

DEFINITION. For an absorbing Markov chain, the fundamental matrix is defined to be $N=(I-Q)^{-1}$.

DEFINITION. The function $n_{j}$ is defined to be the total number of times that the process is in $s^{\cdot}$. (This is defined only for nonabsorbing state $\left.s_{j}\right)$ The function $d_{j}^{(k)}$ is defined to be equal to 1 if the process is in state $\mathrm{s}_{\mathfrak{g}}$ after $\mathrm{k}$ steps, and is 0 otherwise.

It is now possible to give a probabilistic interpretation to $N$. Iet $\left\{s_{n}\right\}$ be the set of nonabsorbing states and $M_{i}\left[n_{j}\right]$ be the mean value of the function $n_{j}$ if the process is started in $s_{i}$.

1.2 THEOREM. $\left\{M_{i}\left[n_{j}\right]\right\}=N_{\text {, where }} s_{i}, s_{j} \in\left\{s_{n}\right\}$ 
$-10-$

PROOF. It is easily seen that

$$
n_{j}=\sum_{k=0}^{\infty} d_{j}^{(k)}
$$

Hence,

$$
\begin{aligned}
& \left\{M_{i}\left[n_{j}\right]\right\}=\left\{M_{1}\left[\sum_{k=0}^{\infty} d_{j}^{(k)}\right]\right\} \\
& =\left\{\sum_{k=0}^{\infty} M_{i}\left[d_{j}^{(k)}\right]\right\} \\
& =\left\{\sum_{k=0}^{\infty}\left(\left(1-p_{i j}^{(k)}\right) \cdot 0+p_{i j}^{(k)} \cdot 1\right)\right\} \\
& =\sum_{k=0}^{\infty}\left\{p_{i j}^{(k)}\right\} \\
& =\sum_{k=0}^{\infty} Q^{k} \text { since } s_{i}, s_{j} \text { are nonabsorbing } \\
& =\mathrm{N}_{\text {. }}
\end{aligned}
$$

This completes the proof ${ }^{3}$ 
This theorem establishes the fact that the mean of the total number of times the process is in a given monabsorbing state is always finite, and that these means are simply given by $\mathrm{N}$.

DEFINITION. Let $t$ be a function giving the number of steps (including the original position) in which the process is in a nonabsorbing state.

If the process starts in an absorbing state, then $t=0$. If the process starts in a nonabsorbing state, then $t$ gives the total number of steps needed to reach an absorbing state. In an absorbing chain, this is the time to absorption.

For the next theorem, it will be assumed that there are s nonabsorbing states and that $\vec{C}$ is an s-component column vector with all. entries equal to 1.

1.3 THEOREM. $\left\{M_{i}[t]\right\}=N \vec{C}$

PROOF. It is easily seen that

$$
t=\sum_{s_{j} \in\left\{s_{n}\right\}} n_{j}
$$

Hence,

$$
\left\{M_{i}[t]\right\}=\left\{M_{i}\left[\sum_{s_{j} \in\left\{S_{n}\right\}} n_{j}\right]\right\}
$$




$$
\begin{aligned}
& =\left\{\sum_{s_{j} \in\left\{S_{n}\right\}} M_{i}\left[n_{j}\right]\right\} \\
& =N \vec{C}
\end{aligned}
$$

since this gives the row sums of $\mathrm{N}$. By summing the rows of $\mathrm{N}$, the mean number of steps the process takes before absorption can be obtained for each starting state $s_{i} \cdot$

For the next theorem, let $\left\{\mathrm{S}_{a}\right\}$ be the set of absorbing states.

1.4 THEOREM $_{0}$ If $b_{i j}$ is the probability that the process starting in nonabsorbing state $s_{i}$ ends up in absorbing state $s_{j}$, then

$$
\left\{b_{i j}\right\}=B=N R, \quad s_{i} \in\left\{s_{n}\right\}, s_{j} \in\left\{s_{a}\right\}
$$

PROOF. Starting in $s_{i}$, the process may be absorbed in $s_{j}$ in one or more steps. The probability of absorption on a single step is $p_{i j}$. If this does not happen, the process may move either to another absorbing state (in which case it is impossible to reach $s_{j}$ ), or to a nonabsorbing state $s_{k^{\circ}}$ In the latter case there is probability $b_{k j}$ of being absorbed in the right state. Hence,

$$
b_{i j}=p_{i j}+\sum_{s_{k} \in\left\{S_{n}\right\}} p_{i k} \cdot b_{k j}
$$


which can be written in matrix form as

$$
\mathrm{B}=\mathrm{R}+\mathrm{QB} \text {. }
$$

Thus,

$$
\begin{aligned}
B & =(I-Q)^{-1} R \\
& =N R .
\end{aligned}
$$

Therefore, by multiplying the $N$ (fundamental) matrix on the right by the $\mathbb{R}$ matrix obtained from the original canonical form, the $B$ matrix of absorption probabilities is obtained.

The review of Markov chain theory is now complete. By using Theorems $1.2,1.3$ and 1.4 , all three questions concerning finite absorbing Markov chains can now be answered. 
Another special class of Markov chains called random walks will now be introduced.

DEFINITION. A random walk is a Markov chain which represents a lattice of states in $n$-dimensional Euclidian space with the property that if the process is in a given state $\mathbf{s}_{\mathbf{k}}$, then in a single step the process can only move to a state immediately adjacent to $\mathrm{s}_{\mathrm{k}}$.

If the set of states in the lattice is finite, the random walk is said to be finite. Recalling the definition of absorbing Markov chains, absorbing random walks may be similarly defined.

DEFINITION. A state in a random walk is an absorbing state if it is impossible to leave it. A random walk is absorbing if (1) it has at least one absorbing state, and (2) from every state it is possible to go to an absorbing state (not necessarily in one step).

The class of random walks that this paper intends to investigate will be the class of finite absorbing random walks. The main question concerning this class of random walks that the resistive analogue method of solution will answer is: What is the probability that the process, if started in nonabsorbing state $s_{i}$, will be absorbed in state $s_{j}$ ? This question may be answered, using Markov chain theory, by solving for the $B$ matrix. However, if there are s number of nonabsorbing states in 
in the lattice, it will necessitate inverting an $s$ X matrix. This $s \times s$ matrix is the (I-Q) matrix constructed from the original canonical form.

To illustrate the classical method of solution, the following simple 2-dimensional random walk will be used. See Fig. 1. The numbered squares indicate the absorbing states and the numbered circles indicate the nonabsorbing states. The lines connecting the states indicate the path that the random walk may take.

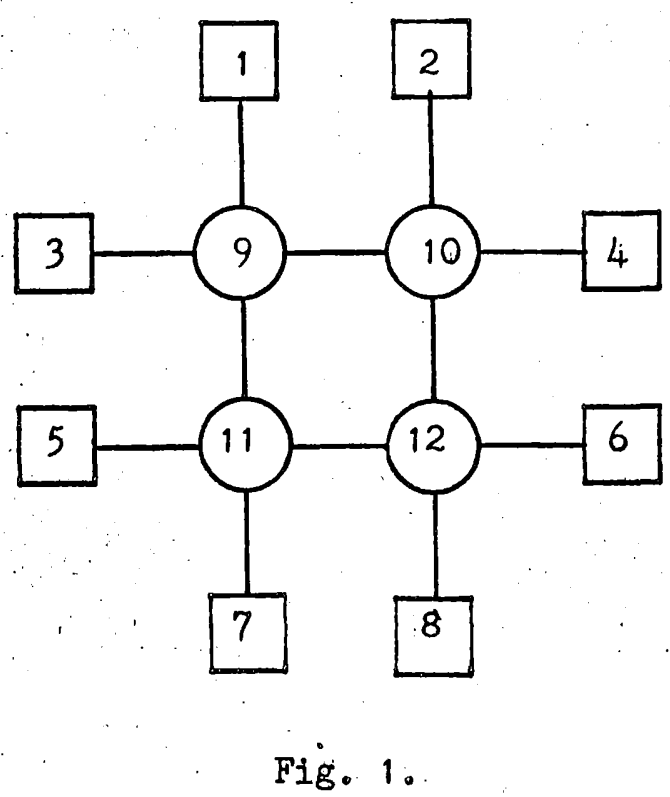

If the probability is $1 / 4$ that the process will take each of the four steps available (assuming that it started in a nonabsorbing state), then the $P$ matrix is 


$$
\begin{aligned}
& \begin{array}{llllllllllll}
1 & 2 & 3 & 4 & 5 & 6 & 7 & 8 & 9 & 10 & 11 & 12
\end{array} \\
& 1\left[\begin{array}{llllllll:llll}
1 & 0 & 0 & 0 & 0 & 0 & 0 & 0 & 0 & 0 & 0 & 0
\end{array}\right] \\
& 2\left[\begin{array}{llllllll|llll}
0 & 1 & 0 & 0 & 0 & 0 & 0 & 0 & 0 & 0 & 0 & 0
\end{array}\right.
\end{aligned}
$$

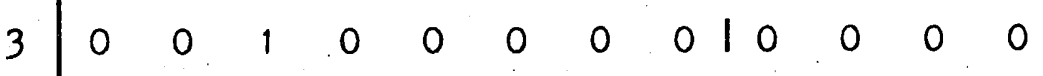

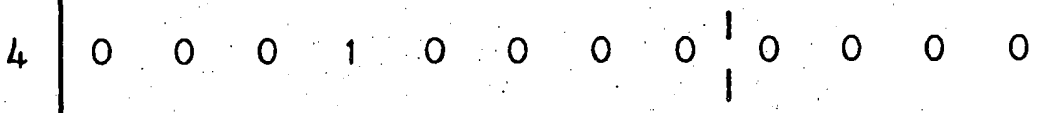

$$
\begin{aligned}
& 5 \quad \begin{array}{lllllllllllll}
5 & 0 & 0 & 0 & 1 & 0 & 0 & 0 & 0 & 0 & 0 & 0 .
\end{array}
\end{aligned}
$$

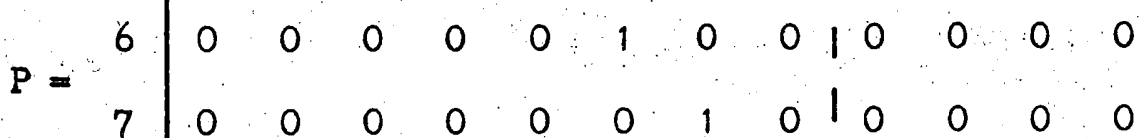

$$
\begin{aligned}
& \begin{array}{lllllllllllllll}
7 & 0 & 0 & 0 & 0 & 0 & 0 & 1 & 0 & 1 & 0 & 0 & 0 & 0
\end{array} \\
& 8{ }_{9} \frac{0}{1 / 4}, \frac{0}{0}-\frac{0}{1 / 4}-\frac{0}{0}-\frac{0}{0}-\frac{0}{0}-\frac{1}{0}+\frac{1}{0}-\frac{0}{1 / 4} \frac{0}{1 / 4}-\frac{0}{0} \\
& 10 \quad \begin{array}{lllllllllllll}
0 & 1 / 4 & 0 & 1 / 4 & 0 & 0 & 0 & 0 & 11 / 4 & 0 & 0 & 1 / 4
\end{array} \\
& \begin{array}{lllllllllllll}
1.1 & 0 & 0 & 0 & 0 & 1 / 4 & 0 & 1 / 4 & 0 & 1 / 1 / 4 & 0 & 0 & 1 / 4
\end{array} \\
& 12\left[\begin{array}{llllllllllll}
0 & 0 & 0 & 0 & 0 & 1 / 4 & 0 & 1 / 4 & 0 & 1 / 4 & 1 / 4 & 0
\end{array}\right]
\end{aligned}
$$

From the canonical form of the $P$ matrix it can be seen that the $Q$ matrix is

$$
Q=10\left[\begin{array}{cccc}
9 & 10 & 11 & 12 \\
0 & 1 / 4 & 1 / 4 & 0 \\
1 / 4 & 0 & 0 & 1 / 4 \\
1 / 4 & 0 & 0 & 1 / 4 \\
0 & 1 / 4 & 1 / 4 & 0
\end{array}\right] .
$$

The (I-Q) matrix is then 


$$
(I-Q)=10\left[\begin{array}{cccc}
9 & 10 & 11 & 12 \\
1 & -1 / 4 & -1 / 4 & 0 \\
-1 / 4 & 1 & 0 & -1 / 4 \\
-1 / 4 & 0 & 1 & -1 / 4 \\
0 & -1 / 4 & -1 / 4 & 1
\end{array}\right] .
$$

The inverse of this (I-Q) matrix is

$$
\begin{aligned}
& \begin{array}{llll}
9 & 10 & 11 & 12
\end{array}
\end{aligned}
$$

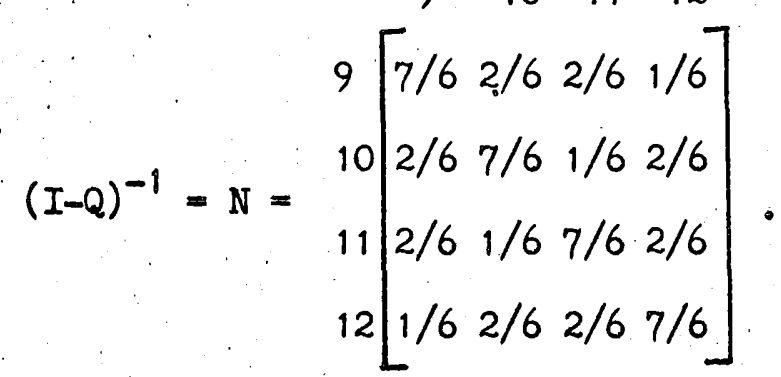

Finally, the B matrix is

$$
\begin{aligned}
& \begin{array}{llllllll}
1 & 2 & 3 & 4 & 5 & 6 & 7 & 8
\end{array} \\
& 9\left[\begin{array}{llllllll}
7 / 24 & 2 / 24 & 7 / 24 & 2 / 24 & 2 / 24 & 1 / 24 & 2 / 24 & 1 / 24
\end{array}\right] \\
& B=N R=10 \quad 2 / 24 \quad 7 / 24 \quad 2 / 24 \quad 7 / 24 \quad 1 / 24 \quad 2 / 24 \quad 1 / 24 \quad 2 / 24 \\
& 11 \quad 2 / 24 \quad 7 / 24 \quad 2 / 24 \quad 1 / 24 \quad 7 / 24 \quad 2 / 24 \quad 7 / 24^{\prime} 2 / 24
\end{aligned}
$$

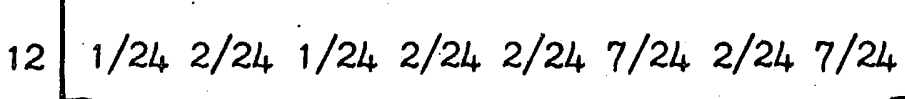


The definition of an electric circuit that this paper will use will be the following one.

DEFINITION. An electric circuit is a finite number of terminals, some of which are connected by wires. The wire connecting terminals $i$ and $j$ has resistance $r_{i j}$ and conductance $g_{i f}=1 / r_{i j}$. If there is no connection between terminals $i$ and $j$, then $g_{i j}=0$; also, $g_{i j}=0$ for all terminals. The voltage on terminal $i$ will be $v_{i}$

To insure that all terminals are connected, it will be assumed that for each terminal i,

(7)

$$
0<\sum_{j} g_{i j}<\infty .
$$

It will now be necessary to differentiate between two different types of terminals. Let $\left\{V_{n}\right\}$ be the set of terminals at which no fixed voltage is applied and let $\left\{\mathrm{V}_{a}\right\}$ be the set of terminals at which fixed voltages of 1 volt or 0 volts (ground) are applied. (These sets are formally equivalent to the sets $\left\{S_{n}\right\}$ and $\left.\left\{S_{a}\right\}_{.}\right)$

Now, as a consequence of Ohm's Law and Kirchoff's Law,

$$
\sum_{k}\left(v_{i}-v_{k}\right) \cdot g_{i k}=0, \quad v_{i} \in\left\{v_{n}\right\} .
$$




$$
\begin{aligned}
& \sum_{k} v_{i} \cdot g_{i k}=\sum_{k} v_{k} \cdot g_{i k} \\
& \sum_{i}=\frac{\sum_{k} v_{k} \cdot g_{i k}}{\sum_{k} g_{i k}}
\end{aligned}
$$

By letting

$$
p_{i j}=\frac{g_{i j}}{\sum_{k} g_{i k}},
$$

then

$$
v_{i}=\sum_{k} p_{i k} \cdot v_{k}
$$

where

$$
\sum_{k} p_{i k}=1 \text { 。 }
$$

In other words, any terminal voltage $v_{i} \in\left\{v_{n}\right\}$ is a weighted average of the neighboring terminal voltages. The $p_{i j}$ terms can be considered as the same transition probability terms of the Markov chain.

If the set of terminals $\left\{\mathrm{v}_{\mathrm{n}}\right\}$ are now considered as nonabsorbing states and the set of terminals $\left\{\mathrm{V}_{\mathrm{a}}\right\}$ are considered as absorbing states, 
then

$$
\vec{V}_{n}=\overrightarrow{R V}_{a}+Q \vec{V}_{n}
$$

where $\vec{V}_{n}$ and $\vec{V}_{a}$ are column vectors and $R$ and $Q$ are the matrices obtained from the canonical form of the transition matrix representing the electric circuit.

1.5 THEOREM. $\quad \overrightarrow{\mathrm{V}}_{\mathrm{n}}=\overrightarrow{\mathrm{BV}}_{\mathrm{a}}$

PROOF. Given the relationship.

$$
\vec{V}_{n}=\overrightarrow{R V}_{a}+Q \vec{V}_{n}
$$

then

$$
\begin{aligned}
\vec{V}_{n}-Q \vec{V}_{n} & =R \vec{V}_{a} \\
(I-Q) \vec{V}_{n} & =\overrightarrow{R V}_{a} \\
\vec{V}_{n} & =(I-Q)^{-1} \overrightarrow{R V}_{a} \\
& =\overrightarrow{N R V}_{a} \\
& =\overrightarrow{B V}_{a}
\end{aligned}
$$


Now, as a consequence of Thèorem 1.5, if

(8) $\quad v_{j}=1$,

(9)

$$
v_{k}=0 \quad \text { for } j \neq k \text { and } v_{j}, v_{k} \in\left\{v_{a}\right\}
$$

then,

$$
\left(b_{1 j}, b_{2 j}, \ldots, b_{s j}\right)=\vec{v}_{n}^{\top}
$$

This is accomplished electrically by putting 1 volt on terminal $j$ and 0 volts (ground) on the rest of $\operatorname{set}\left\{V_{a}\right\}$.

A resistive analogue will be defined in the following manner.

DEFINITION. A resistive analogue is that electric circuit which is equivalent to a given finite absorbing random walk.

Another definition that will be needed is the following one.

DEFINITION. For any state $s_{i} \in\left\{S_{n}\right\}$ in a finite absorbing random walk, the set of transition probabilities $\left\{p_{i k}\right\}$ will be defined as the probability profile of state $s_{i}$ : 
Whether or not a finite absorbing random walk has a resistive analogue is determined by its probabilities profiles. Since any conductance $g_{i j}$ not only determines $p_{i j}$ but also $p_{j i}$, and conductances are bidirectional, $g_{i j}=g_{j i}$ must be a mathematical requirement for all $s_{i}, s_{j} \in\left\{s_{n}\right\}$.

1.6 THEOREM. If a finite absorbing random walk has a probability profile of $\left\{p_{i k}\right\}=p_{i k}=1 / m, k=1,2, \ldots, m$ for all $s_{i} \in\left\{s_{n}\right\}$, then the random walk has a resistive analogue.

PROOF. By definition,

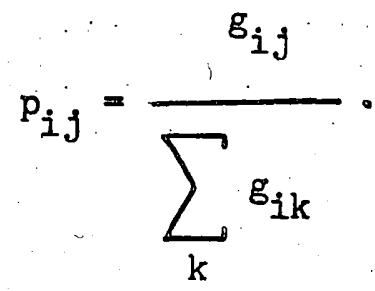

Now, if $\lambda$ is defined as

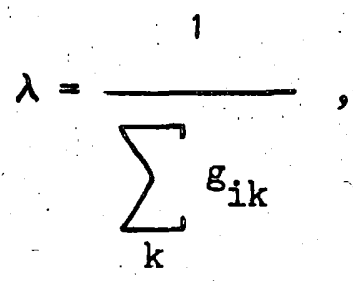

then

$$
p_{i j}=\lambda\left(g_{i j}\right) .
$$


But,

$$
p_{i 1}=p_{i 2}=\cdots=p_{i m}
$$

Therefore,

$$
\begin{aligned}
\lambda g_{i 1} & =\lambda g_{i 2}=\cdots=\lambda g_{i m} \\
g_{i 1} & =g_{i 2}=\cdots=g_{i m} \\
g_{i j} & =g_{j i}
\end{aligned}
$$

1.7 THEOREM. A constant multiple of all conductances will not change any of the probability profiles.

PROOF. From Theorem 1.6,

$$
p_{i j}=\lambda\left(g_{i j}\right)
$$

However,

$$
\begin{aligned}
\frac{\lambda\left(g_{i j}\right)}{\sum_{k} \lambda\left(g_{i k}\right)}=\frac{\lambda\left(g_{i j}\right)}{\lambda \sum_{k} g_{i k}} \\
=\frac{g_{i j}}{\sum_{k} g_{i k}}=p_{i j} \quad \text { for any } \lambda \neq 0 .
\end{aligned}
$$


1.8 THEOREM. Two resistive analogues are equivalent if one is a constant multiple of the other.

PROOF. This theorem follows from Theorem 1.6 and Theorem 1.7.

To sum up the procedure thus far, if a finite absorbing random walk has a constant probability profile (satisfies Theorem 1.6), then a set of conductances $\left\{g_{i j}\right\}$ may be substituted for its set of transition probabilities $\left\{p_{1 j}\right\}$. Further, these conductances can be any value as long as they are all the same. (Of course if they are too high, the current demand on the 1 volt power supply may become excessive.) Then, by applying 1 volt to a particular absorbing terminal $s_{j}$ and grounding the rest of the absorbing terminals, the voltage on any particular nonabsorbing terminal $s_{i}$ will equal the absorption probability $b_{i f}$ for the random walk that the resistive analogue represents. 
The first random walk that was analyzed is the triangular shaped one pictured in Fig. 2 .

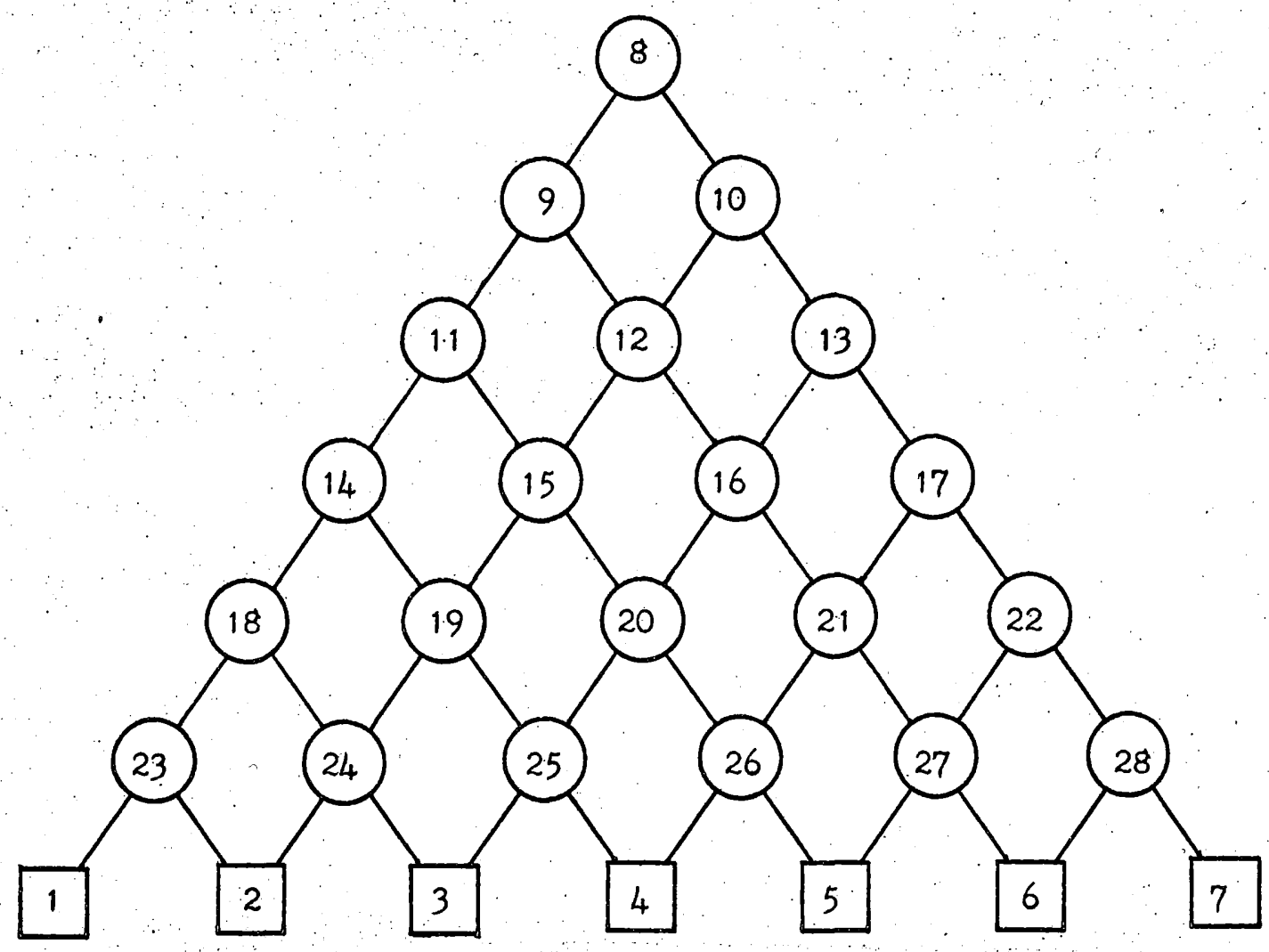

Fig. 2 .

There are 21 nonabsorbing states (28 states minus 7 absorbing states) in this particular random walk. To determine the various absorption probabilities would involve solving a $21 \times 21$ system of linear equations.

The set of probabilities the writer was interested in is the set obtained if the process is started in state 8. If the process is 
started in state 8 and allowed to move only from top-to-bottom, the distribution in absorbing states 1 through 7 is the familiar binomial one. However, if the process is started in state 8 and allowed to move in a random walk fashion, will the distribution in absorbing states 1 through 7 still be binomial?

The answer is no. By allowing the process to be a random walk, the probability of absorption on the end states such as 1, 2, 6 and 7 will be higher than in the binomial distribution. Thus, the random walk tends to diffuse the process to the end states.

The absorption probabilities in the seven states (if the process is started in state 8) are:

\section{State}

1 2

3

4 5

6

7

\section{Probability}

.046

.134

.204

.232

.204

.134

.046

$$
1.000
$$

Because of Theorem 1.0, the sum of the absorption probabilities must be 1. As can be seen, the accuracy appears to be quite good. It is unlikely that the individual probabilities differ from their true 
values by more than .001 .

Fig. 3 shows the graph of both the random walk and binomial absorption probabilities.

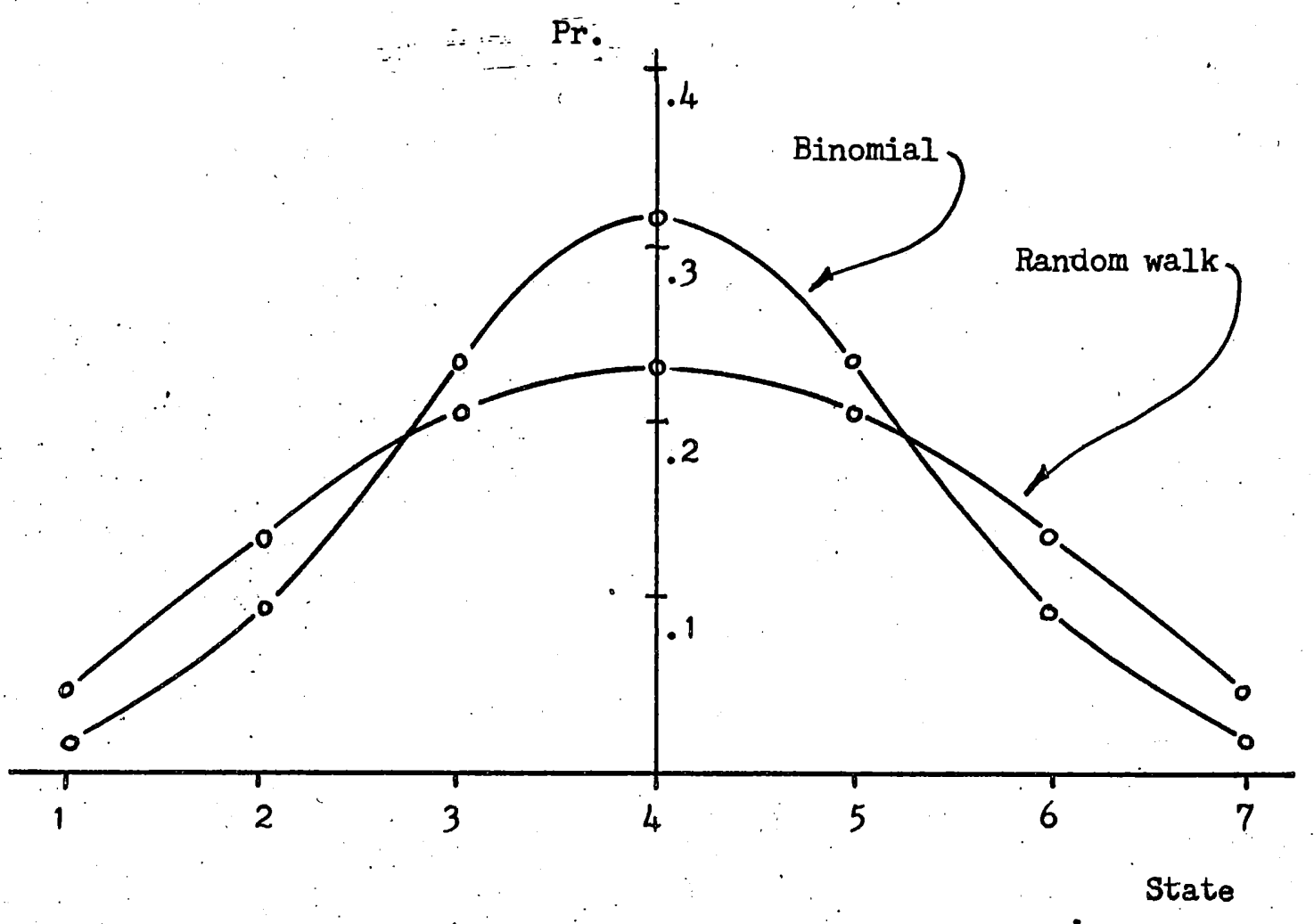

Fig. 3.

The complete solutions are graphically portrayed in Fig. 4, Fig. 5, Fig. 6 and Fig. 7.' The absorbing state in question has an " $x$ " marked in it while the corresponding absorption probabilities are listed in their respective nonabsorbing state circles. 


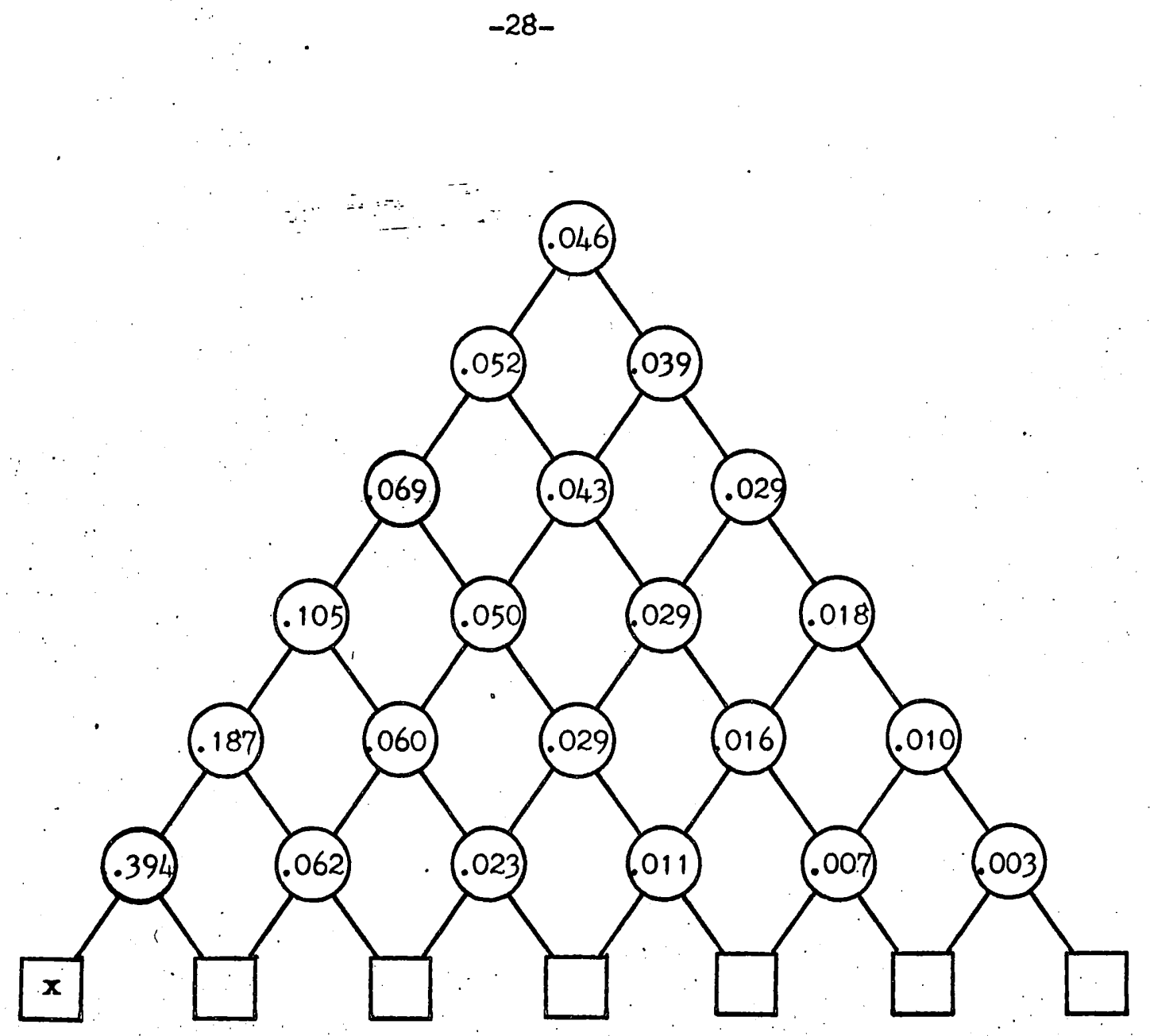

Fig. 4 . 


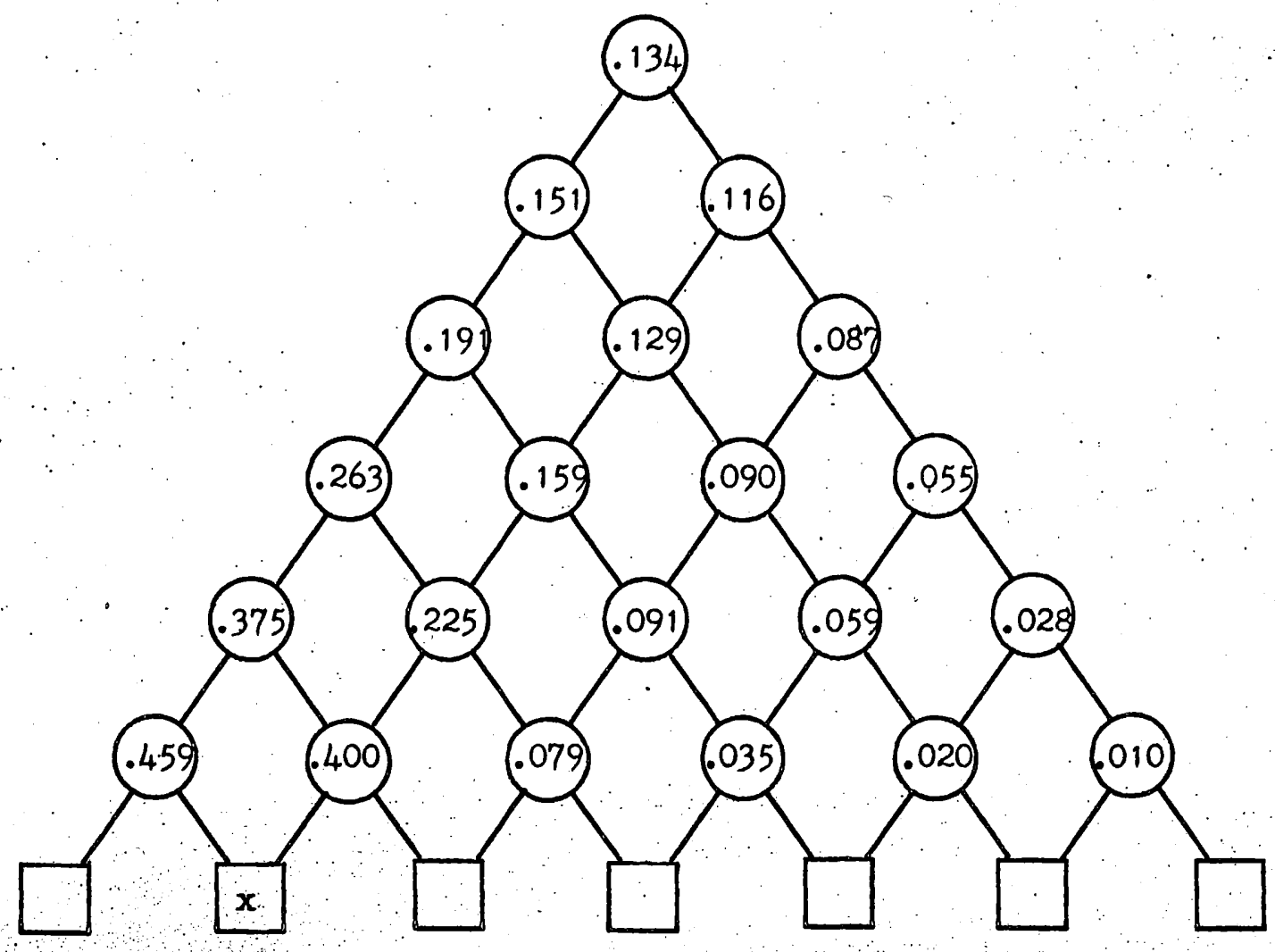

Fig. 5. 


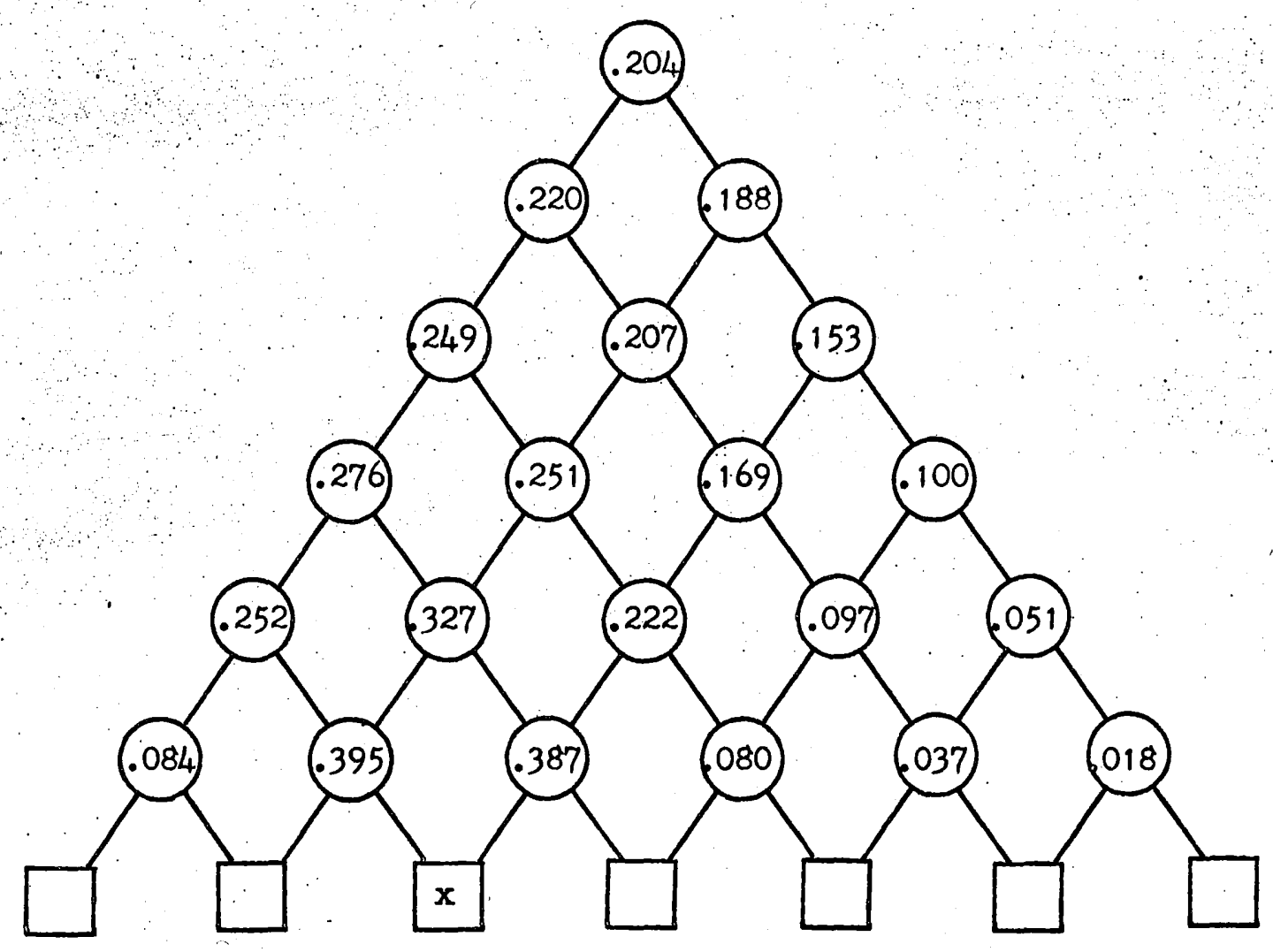

Fig. 6 . 


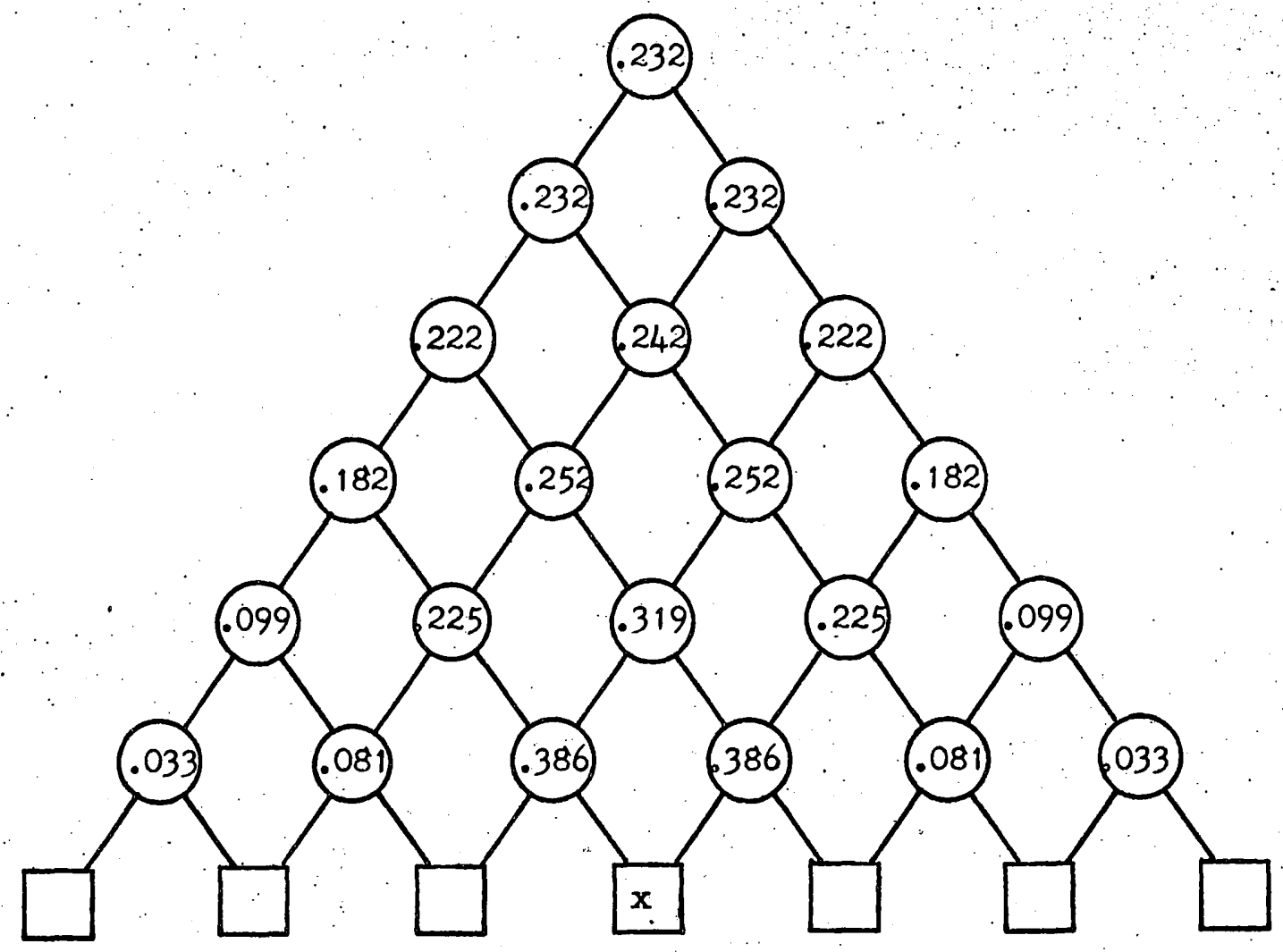

Fig. 7.

Because of the center-fold symmetry in Fig. 7, many of the absorption probabilities are redundant. Also, the absorption probabilities of states 1,2 and 3 are identical to those of states 5,6 and 7 respectively. For this reason, only four diagrams are needed for the complete solution.

Many times, due to symmetry, solution sets of absorption probabilities are identical for different absorbing states. While the triangular shaped random walk with seven absorbing states has four dis- 
tinctly different solution sets, the circular shaped random walk in Fig. 8 with eight absorbing states has only one distinctly different solution set.

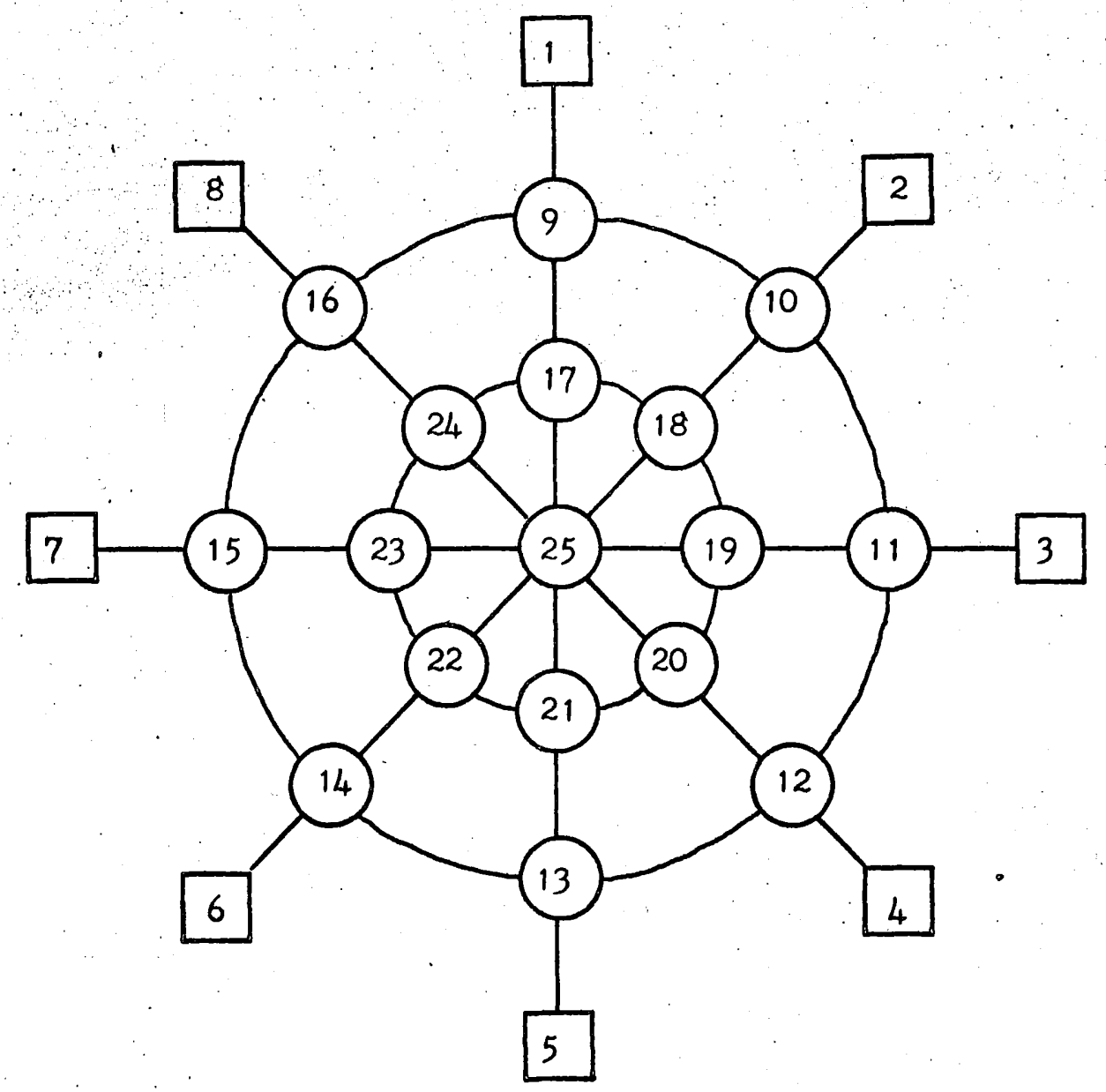

Fig. 8.

In this particular random walk, there are 17 nonabsorbing states. Thus, a formal solution of the various absorption probabilities would require solving a $17 \times 17$ system of linear equations. 
If the process is started in state 25 , the random walk represents a finite "drunkard's walk." However, by starting the process in state 25 , the absorption probabilities are $1 / 8$ that the process will be absorbed in any one particular absorbing state and as such are trivial. Much more interesting is the case where the process is started in a nonabsorbing state other than state 25. The complete solution is graphically portrayed in Fig. 9.

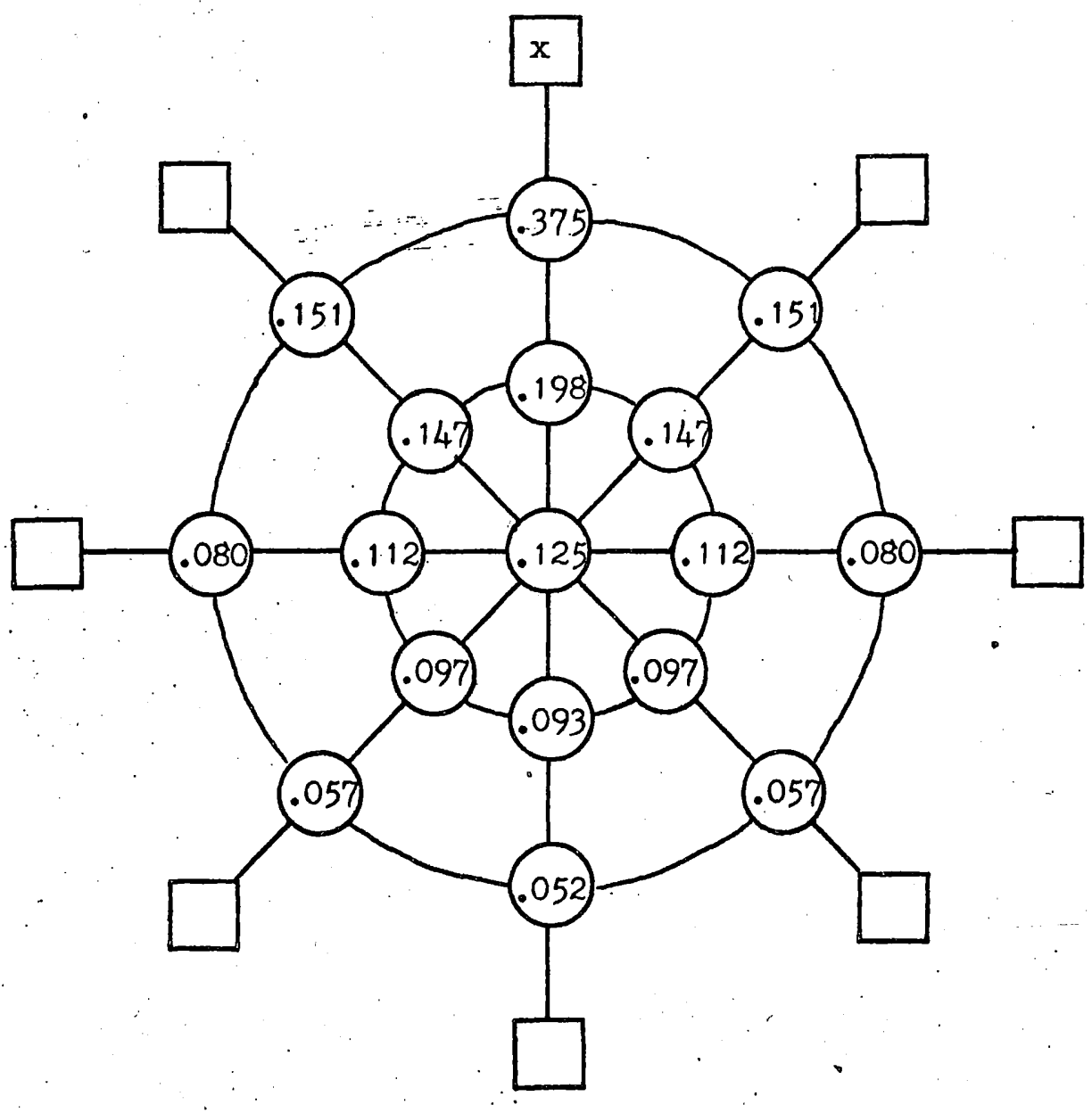

Fig.9. 
This random walk also has center-fold symmetry and as such, many of the absorption probabilities are redundant. A more interesting property is that the sum of the absorption probabilities in a ring is 1. This property is a consequence of Theorem 1.0 and four-fold symmetry. For example, Theorem 1.0. states that if the process is started in state 9, the sum of the absorption probabilities of being absorbed in states 1 through 8 is 1. However, due to symmetry, the probability of starting in state 9 and being absorbed in states 8 and 2 is identical to the probability of starting in states 10 and 16 and being absorbed in state: 1 . To continue around the ring, new notation will now be introduced.

Recalling that $b_{i j}$ is the probability that the process starting in nonabsorbing state $s_{i}$ ends up in absorbing state $s_{j}$, let

$$
b_{i j}=\operatorname{Pr}\left[s_{i} \stackrel{a}{\rightarrow} s_{j}\right]
$$

Now, as a consequence of the four-fold symmetry,

$$
\begin{aligned}
& \operatorname{Pr}[9 \stackrel{a}{\rightarrow} 2,8]=\operatorname{Pr}[10,16 \stackrel{a}{\rightarrow} 1] \\
& \operatorname{Pr}[9 \stackrel{a}{\longrightarrow}, 3,7]=\operatorname{Pr}[11,15 \stackrel{a}{\rightarrow} 1] \\
& \operatorname{Pr}[9 \stackrel{a}{\longrightarrow} 4,6]=\operatorname{Pr}[12,14 \stackrel{a}{\longrightarrow} 1] \\
& \operatorname{Pr}[9 \stackrel{a}{\longrightarrow} 5]=\operatorname{Pr}[13 \stackrel{a}{\rightarrow} 1]
\end{aligned}
$$

The same argument also applies to the inner ring that was just applied to the outer ring. The sums of these rings are: 
Inner ring

$$
\begin{aligned}
& .198 \\
& .147 \\
& .112 \\
& .097 \\
& .093 \\
& .097 \\
& .112 \\
& .147 \\
& \hline 1.003
\end{aligned}
$$

Outer ring .375 .151

.080

.057

.052

.057

.080

.151

1.003

The third and final random walk that was analyzed is the square shaped one pictured in Fig. 10. 


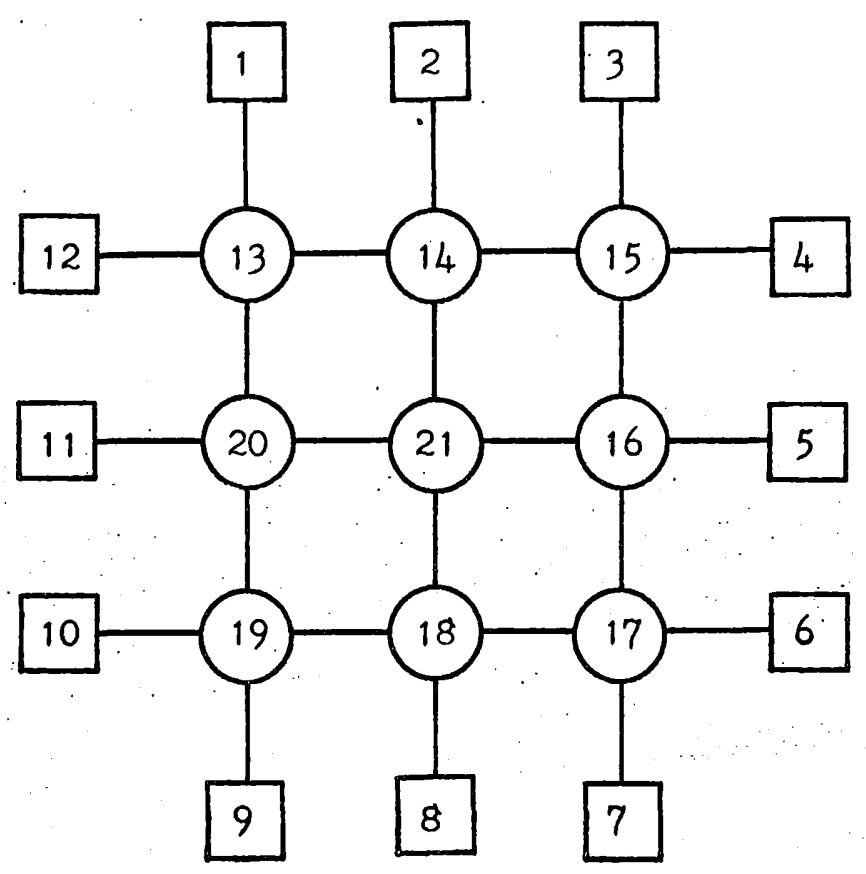

Fig. 10。

In this random walk, although there are 12 absorbing states, only two diagrams are needed for the complete solution. These two solutions are graphically portrayed in Fig. 11 and Fig. 12. 


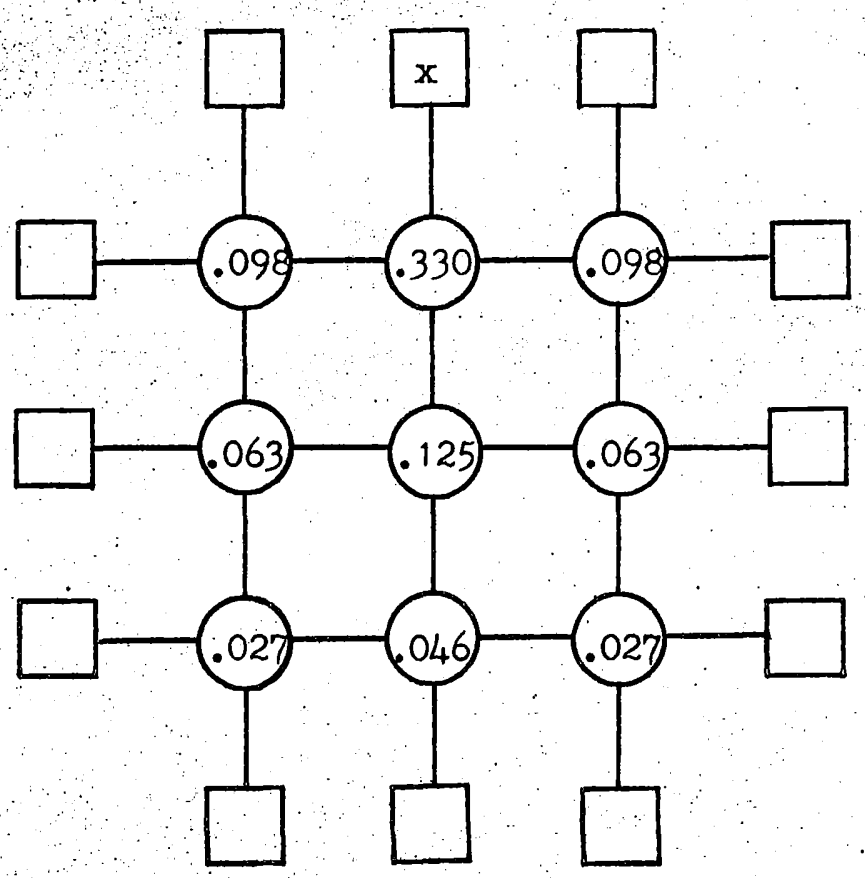

Fig. 11. 


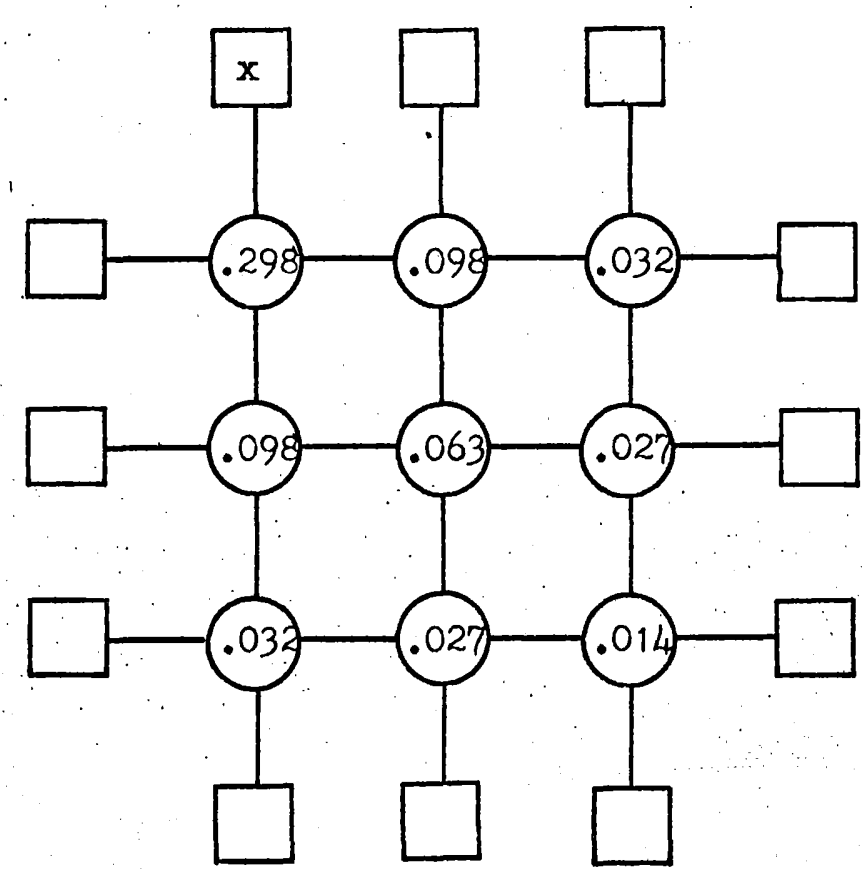

Fig. 12.

Once again, symmetries produce redundancies in the absorption probabilities. The diagram in Fig. 11 has center-fold symmetry while the diagram in Fig. 12 has diagonal-fold symmetry. In both cases, the nonredundant probabilities lie on the line or axis of symmetry. There are probably many other properties that could be pursued in connection with symmetry in random walks; but the writer believed most of them would be outside the scope of this paper.

The main purpose of this paper is to demonstrate an alternate method for solving absorption probabilities of random walks -- as opposed to using a digital computer. However, the last random walk was solved both ways for comparison of accuracy. By rounding the 
computer results to three places, the absorption probabilities (if the process is started in. state 13) are:

State $\quad$ Resistive analogues Digital computer

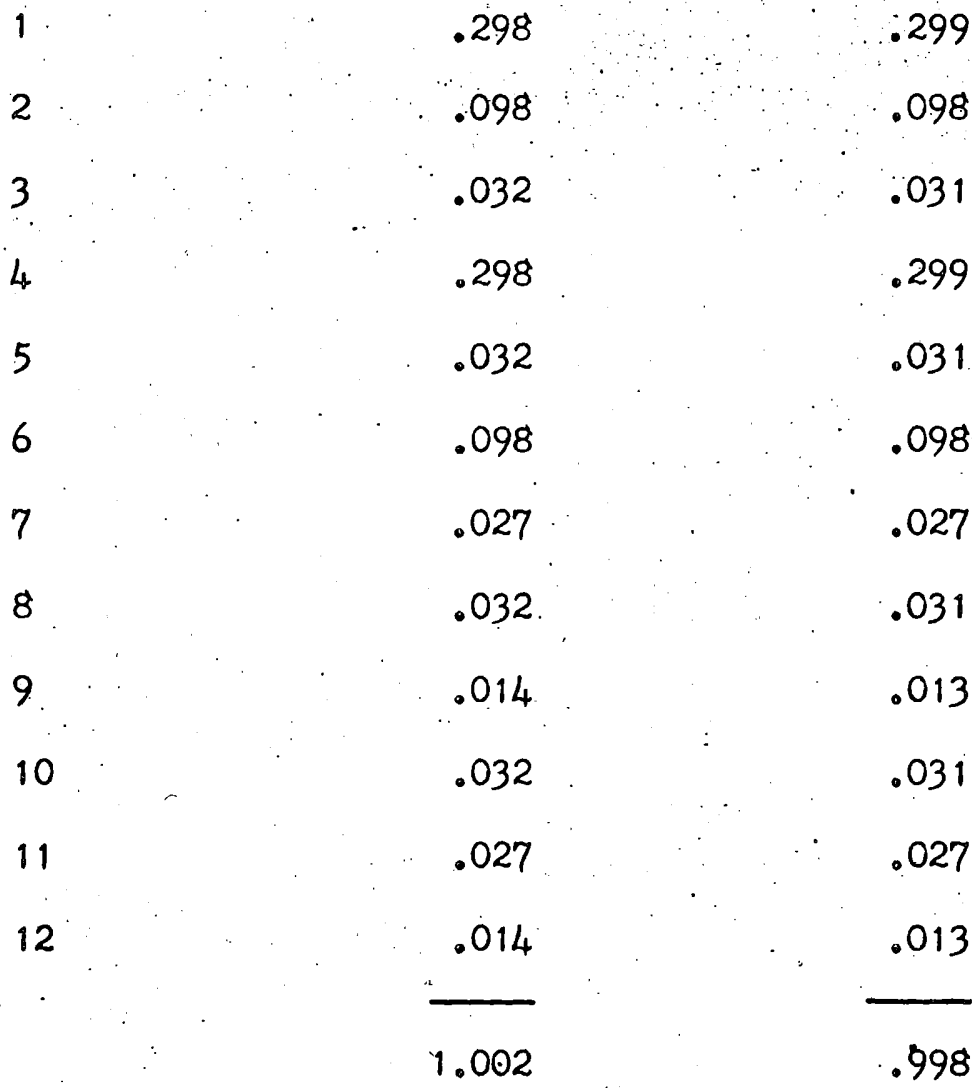

The correlation between the two methods is quite good. By rounding the digital computer results, the three-place accuracy never differs by more than .001 . One reason for this accuracy was the use of $1 \%$ precision resistors and a digital readout voltmeter that was also $1 \%$ accurate. The resistors were $1.024 \mathrm{k} \Omega$ in value and were industrial castoffs because their peculiar value was no longer needed. The writer would encourage obtaining resistors in this fashion be- 
cause of the lack of expense in obtaining a large number of precision resistors that have the same value of resistance.

Wiring the resistors is best accomplished by soldering them on the heads of nails that have been driven into wood. This method provides a rigid model that can also be used for classroom demonstrations. If time is limited, a breadboard fashion of connecting the resistors would probably be best. In either case, the resistive analogue method does provide a useful alternative method of solving absorbing random walk problems. 
Doob, J. I., Stochastic Processes, New York, John Wiley \& Sons, Inc., (1953).

Karlin, S., and McGregor, J., "Random walks," Illinois J. Math., 3, $66-81,(1959)$.

1 Kemeny, J. G., Mirkil, H., Snell, J. L., and Thompson, G. L., Finite Mathematical Structures, Englewood Cliffs, N. J., Prentice-Hall, Inc., (1959)。

2 Kemeny, J. G., and Snell, J. I., Finite Markov Chains, Princeton, N. J., D. Van Nostrand Co., Inc., (1960).

3 Kemeny, J. G., Snell, J. L., and Knapp, A. W., Denumerable Markov Chains, Princeton, N. J., D. Van Nostrand Co., Inc., (1966). Nash-Williams, C. St J. A., "Random walk and electric currents in networks,"! Proc. Cambridge Phil. Soc., 55, 181-194, (1959). Parzen, E., Stochastic Processes, San Francisco, Holden-Day, Inc., (1962).

Spitzer, F. L., Principles of Random Walk, Princeton, N. J., D. Van Nostrand Co., Inc., (1964).

Takacs, I., trans. by Zador, P., Stochastic Processes, New York, John Wiley \& Sons, Inc., (1960).

Wax, $\left(e_{0}\right)$, Selected Papers on Noise and Stochastic Processes, New York, Dover Publications, Inc, (1954). 
$p_{i j}$ - Transition probability of moving from state $s_{i}$ to state $s_{j} \cdot$

$p_{i j}^{(n)}-$ Probability of being in state $s_{j}$, starting from state $s_{i}$, after n steps.

$b_{i j}$ - Probability that the process starting in nonabsorbing state $s_{i}$ ends up in absorbing state $s_{j}$.

$r_{i j}$ - Resistance connected between terminal $i$ and terminal $j$.

$g_{i j}$ - Conductance connected between terminal $i$ and terminal $j ;$ and $g_{i j}=1 / r_{i j}$.

P - Transition matrix of transition probabilities.

Q - Partitioned transition matrix containing all nonabsorbing states.

$N$ - Fundamental matrix; and $N=(I-Q)^{-1}$

B - Matrix of absorption probabilities.

$\vec{C}$ - Column vector with all entries equal to 1 . 
$d_{j}^{(k)}$ - Function defined to be 1 if the process is in state $s_{j}$ after $k$ steps, and 0 otherwise.

$n_{j}-$ Function defined to be the total number of times the process is in state $s_{j} \cdot$

$M_{i}\left[n_{j}\right]$ - Mean value of the function $n_{j}$ if the process is started in state $s_{i}$

$\left\{S_{n}\right\}$ - Set of nonabsorbing states.

$\left\{s_{a}\right\}$ - Set of absorbing states.

$\left\{v_{n}\right\}$ - Set of terminals at which no fixed voltage is applied.

$\left\{v_{a}\right\}$ - Set of terminals at which fixed voltages of 1 volt or 0 volts are applied.

$\operatorname{Pr}\left[s_{i} \stackrel{a}{\rightarrow} s_{j}\right]-$ Equivalent to $b_{i j}$ 